\title{
The current issues and future perspective of artificial intelligence for developing new treatment strategy in non-small cell lung cancer: harmonization of molecular cancer biology and artificial intelligence
}

\author{
Ichidai Tanaka ${ }^{1 *} \mathbb{B}$, Taiki Furukawa ${ }^{2}$ and Masahiro Morise ${ }^{1}$
}

\begin{abstract}
Comprehensive analysis of omics data, such as genome, transcriptome, proteome, metabolome, and interactome, is a crucial technique for elucidating the complex mechanism of cancer onset and progression. Recently, a variety of new findings have been reported based on multi-omics analysis in combination with various clinical information. However, integrated analysis of multi-omics data is extremely labor intensive, making the development of new analysis technology indispensable. Artificial intelligence (Al), which has been under development in recent years, is quickly becoming an effective approach to reduce the labor involved in analyzing large amounts of complex data and to obtain valuable information that is often overlooked in manual analysis and experiments. The use of Al, such as machine learning approaches and deep learning systems, allows for the efficient analysis of massive omics data combined with accurate clinical information and can lead to comprehensive predictive models that will be desirable for further developing individual treatment strategies of immunotherapy and molecular target therapy. Here, we aim to review the potential of $\mathrm{Al}$ in the integrated analysis of omics data and clinical information with a special focus on recent advances in the discovery of new biomarkers and the future direction of personalized medicine in non-small lung cancer.
\end{abstract}

Keywords: Artificial intelligence, NSCLC

\section{Introduction}

To improve prognosis of cancer patients, there is a growing trend to analyze numerous types of omics data, such as DNA, RNA, microRNA, protein, and metabolites [1, 2]. Many researchers have been aiming to develop identified markers for clinical application of early cancer detection, prognosis prediction, and evaluation of treatment

*Correspondence: ichidai@med.nagoya-u.ac.jp

${ }^{1}$ Department of Respiratory Medicine, Nagoya University Graduate School of Medicine, 65 Tsurumai-cho, Showa-ku, Nagoya 466-8550, Japan

Full list of author information is available at the end of the article efficacy. The recent advent of next generation sequencing (NGS) has permitted the generation of comprehensive profiles of somatic mutations in various cancer types and has contributed to the rapid advancements made in the field of cancer research. Genome sequence-based studies of large numbers of clinical samples, such as those available through The Cancer Genome Atlas (TCGA) and International Cancer Genome Consortium (ICGA), have led to the identification of a variety of driver gene mutations and oncogenic signaling pathways that give cancer cells a fundamental growth advantage during their neoplastic transformation [3-7]. These studies have original author(s) and the source, provide a link to the Creative Commons licence, and indicate if changes were made. The images or other third party material in this article are included in the article's Creative Commons licence, unless indicated otherwise in a credit line to the material. If material is not included in the article's Creative Commons licence and your intended use is not permitted by statutory regulation or exceeds the permitted use, you will need to obtain permission directly from the copyright holder. To view a copy of this licence, visit http://creativecommons.org/licenses/by/4.0/. The Creative Commons Public Domain Dedication waiver (http://creativeco mmons.org/publicdomain/zero/1.0/) applies to the data made available in this article, unless otherwise stated in a credit line to the data. 
revealed significant genomic heterogeneity, not only in different regions of the same patient, but also within a single tumor, and are contributing to the elucidation of the essential qualities of cancer development and progression [8]. Furthermore, numerous biological networks of genetic mutations affecting DNA copy number, methylation, the proteome, and the transcriptome have been dramatically demonstrated in cancer systems [9-12]. In addition, the recent advanced omics-technologies allow us to conduct single cell multi-omics sequencing, which can characterize the unique genotype and phenotype of each individual cell. This approach can provide new insights into tumor heterogeneity and deep characterization of the tumor microenvironment at a single-cell resolution [13]. Therefore, integration of these diverse omics data with highly accurate clinical information should lead to new clinical developments regarding the prevention of cancer onset and new treatment strategies based on intratumoral heterogeneity.

In parallel with the development of omics data analysis, recent exploitation of artificial intelligence (AI)based technology has progressed rapidly. The theory of AI itself has existed since around the time of World War II, but several endeavors at developing AI failed due to problems associated with the lack of computing power. However, the application of AI in molecular biology has become more common with the advancements in computer technology. In accordance with the development of AI technology, trained deep learning has gradually evolved and currently plays an important role in clinical applications, especially in analyzing radiographs [14-16] and pathological images [17-19]. Meanwhile, the machine learning approach remains to be used mainly for omics data analysis due to the features of small sample sizes and large dimension data [20]. Multiple omics databases, such as TCGA and ICGA, have been dramatically expanded. Additionally, recent new multilayer omics analyses, such as single-cell sequencing, have been generating an extremely huge amount of data, resulting in the rapid evaluation of these massive amounts of data being beyond the capabilities of manual analysis. To reduce the level of labor involved in analyzing huge amounts of complex omics data, successful collaborations between biologists and computer scientists are required. Ultimately, machine learning approaches will play a central role in the creation of efficient strategies for promoting positive cancer research outcomes.

Among the numerous types of cancer, lung cancer can be a pervasive disease that is commonly diagnosed at advanced stages, with non-small-cell lung cancer (NSCLC) being the most prevalent form of lung cancer [21]. In recent decades, two innovative treatment strategies have been established to achieve long-term survival of patients with advanced NSCLC. The first one was based on the discovery of druggable oncogenic driver mutations or fusions. The second was the development of immune oncology, which is especially represented by immune checkpoint inhibitors (ICIs). Pivotal clinical trials have led to the establishment of a variety of first-line therapies as standard treatment strategies for subgroups of patients with NSCLC based on oncogenic driver mutation status and programmed death-ligand 1 (PD-L1) tumor proportion scores [22-25]. Furthermore, various clinical trials investigating new compounds or combination therapies with existing antineoplastic agents have been performed or are ongoing for each subgroup of NSCLC. Unfortunately, primary and acquired resistance against new strategies are a relevant issue and a primary concern as resistance complicates the decision of choosing the best therapeutic strategy among the numerous treatment options available. Therefore, establishment of AI-based comprehensive predictive models for efficacy and toxicity of each treatment is particularly desirable in terms of further developing individual treatment strategies. In this review, we summarize recent medical applications of AI for the analysis of omics data in combination with clinical information for NSCLC and discuss future application of this magnificent and powerful technology to clinical fields.

\section{Al in medicine-concepts and utilization Classification of Al}

According to the algorithm used, AI is categorized as "rule-based," which is called AI in a broad sense, and "non-rule-based," which is referred to as machine learning. For rule-based algorithms, a person provides conditional branches and rules to solve for an optimal answer. For example, if a person defines the AI algorithm with the condition that "when study number of two databases are the same, they are regarded as duplicates and should be integrated," the algorithm will be fully faithful to the command and integrate the numbers. A rule-based algorithm is effective in limited situations in which there are limited choices. However, it is difficult to create a rulebased algorithm under complicated situations.

In contrast, machine learning automatically generates rules from known training data and applies them to the machine-learning algorithm using statistical analysis. Therefore, machine learning is focused on reading patterns from a large amount of data in a short amount of time and can semi-automatically obtain more accurate results than that of manual human evaluations. Machine learning is classified into three types, supervised learning, unsupervised learning, and reinforcement learning $[26,27]$. Supervised learning is a technique in which the learner parameter is updated in order to get closer 
to the correct output. In other words, training data are provided to the algorithm, the correct answer label is learned, and a learning algorithm is generated in which the output is the correct answer label. Next, it is verified whether a value close to the "correct label" is obtained when unknown data is applied to the generated model. This type of machine learning is usually used for classification tasks or regression tasks in image recognition. For example, when a whole slide image of lung cancer is provided as an input and the output is labeled as "normal lung", the learner will be updated by the teacher that the correct answer is "lung cancer" [28]. Supervised learning requires an extensive amount of training data and the following labeled data, which are often difficult to obtain in medical and biological fields. Meanwhile, unsupervised learning is another machine learning technique in which the learner is updated using only inputs without "correct answer" data. Reinforcement learning is the final machine learning technique, and updates the learner through trial and error in order to determine the best course of action to suit the current situation.

Deep learning is a machine-learning technique inspired by the human brain that uses large mathematical functions with millions of parameters based on a neural network structure that combines multiple layers of artificial nerve cells [16]. Using a deep-learning system, great power can be exerted for the recognition and classification of various medical images and is applicable to pathological diagnosis and cancer detection using computed tomography (CT) images [15, 29-31]. While most of deep-learning algorithms to date have been applied using supervised-learning methods to learn a specialist's thought or technique, some deep-learning algorithms have been recently created using unsupervised-learning methods. For instance, Yamamoto et al. developed a deep-learning algorithm that enables an automated acquisition of explainable features from diagnostic annotation-free histopathology images of prostate cancer and identified a new feature that improves the accuracy of diagnosis of prostate cancer recurrence [32]. Essentially, they created a deep-learning algorithm that uses histopathological images of prostate cancer as inputs, which then automatically outputs feature maps of the histopathological images.

\section{Application of Al for analysis of omics data and clinical information}

The application of AI in medicine is currently of great interest, especially in the diagnostic and predictive assessment of medical images [33]. Among AI algorithms, machine learning is able to learn health trajectory patterns from vast numbers of patients (Fig. 1). This can help physicians anticipate future events at an expert level and draw curves from extensive amounts of clinical information, providing insight well beyond the experience from an individual physician's practice. Development of an algorithm for medical diagnosis or prediction typically requires a huge dataset, often referred to as "big data," especially an algorithm in which supervised learning of deep neural networks are used. Accurate algorithms require high quality datasets; however, these big datasets need to be collected in various ways from multiple heterogeneous sources [34]. When the algorithm diagnosis outputs in the training phase differ from the actual diagnosis, the calculated parameter weights are updated in order for the output to approach the correct disease label. This process is then repeated many times. During the updating process, deep learning generally requires an extremely large number of samples to approach the correct answer as the algorithm parameter may exceed one hundred million.

Although the samples available for omics data analysis have been usually limited, deep-structured learning usually requires an extremely large number of samples. Therefore, machine-learning models have been commonly utilized to create quicker and more accurate algorithms under current situations for the analysis of omics data (Fig. 1). Because the inclusion of too much features may lead to overfitting and increases calculation costs, each analysis initially starts with biomarker selection and knowledge of omics data, as well as selection of statistical methods that increase the stability of the feature selection process. In biomedical analysis, the term "features" indicates measured characteristics used as learning input, such as age, gender, or $\mathrm{X}$ genes.

After feature selection, machine learning can be used to achieve various ends, such as disease type or severity classification or mortality prediction. To analyze omics data using machine learning, feature selection is one of the most important procedures because of its large dimension. Interestingly, machine-learning techniques have sometimes been used in the feature selection procedure itself [35]. Best et al. selected specific spliced-RNA biomarker panels using likelihood ratio analysis of variance (ANOVA) statistics and then comparing healthy individuals to patients with cancer based on analysis of differential expression of spliced junctions [36]. Logistic regression analysis, ANOVA statistics, and an ensemble approach with random sub-sampling have been widely used to select important features [37-39]. Another way to reduce the dimension of potential features is using unsupervised-machine learning, such as least absolute shrinkage and selection operator (LASSO) regression or principal component analysis (PCA) [40]. LASSO regression, one approach of regression analysis, has the feature of part of the coefficient being set to zero, 


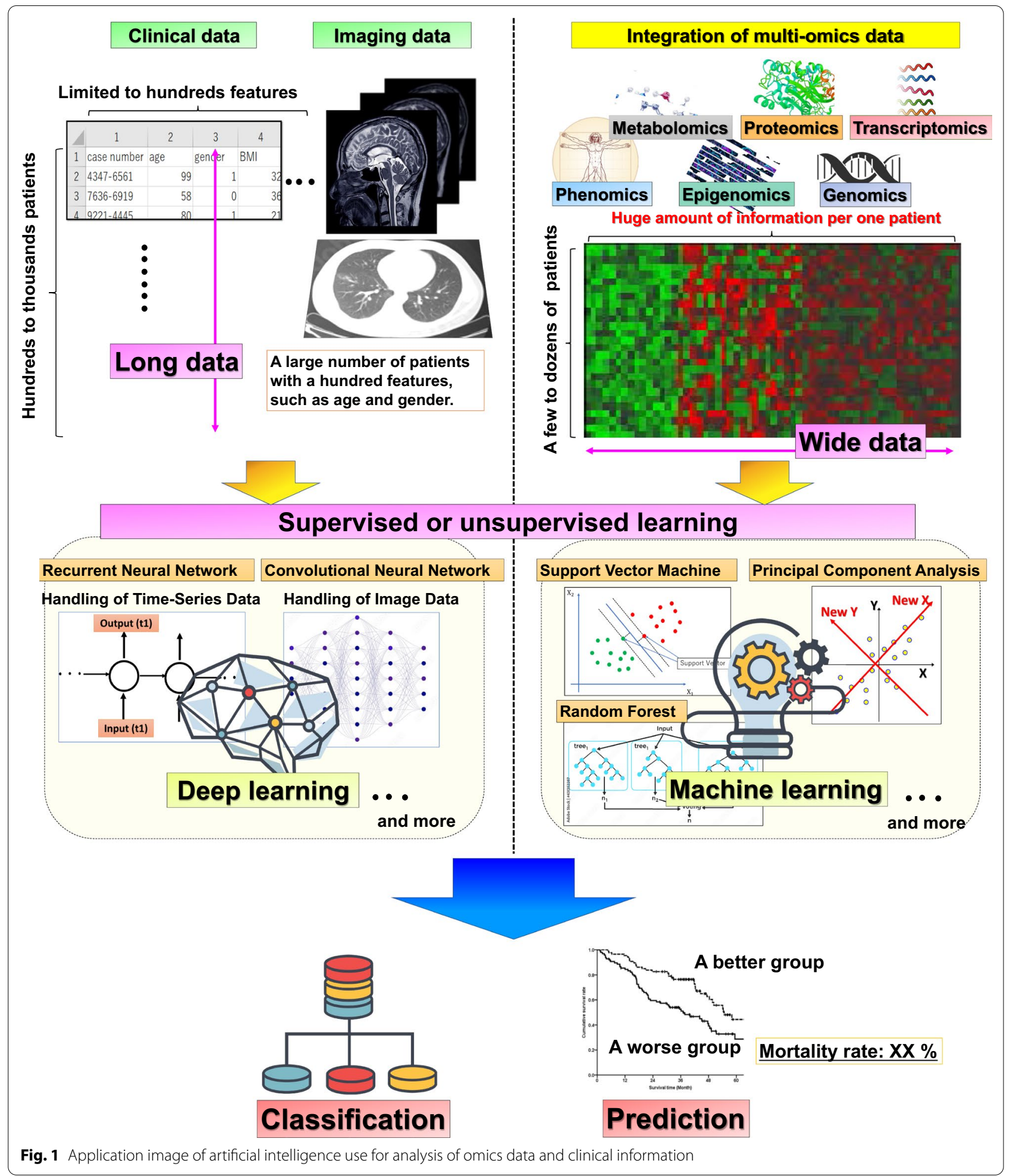

reducing that dimension of the feature. Lu et al. obtained 2139 genetic mutations for consideration in their initial model to predict long-term clinical benefit, which was then reduced using the LASSO model to 161 genetic mutations without a reduction in the clinical prediction accuracy [31]. Meanwhile, PCA weighs and integrates many features to create a relatively small number of new features that represent the overall variability. Guan et al. 
performed PCA to distinguish patients with inflammatory bowel disease (IBD) from control subjects using 55 lipid species [40]. They defined two new features of principal component $1(\mathrm{PC} 1)$ and principal component 2 (PC2), which were able to distinguish between patients with IBD and the control subjects. These filtering steps improve data normalization, which is a critical step in biological data analysis.

Generally, supervised and/or unsupervised learning models, including LASSO, support vector machine (SVM), random forest, and gradient boosting, have been used after feature selection to perform the classification task, such as identifying patients with significantly worse mortality rates. SVM is an algorithm that minimizes the distance of prediction error and is one of the most frequently used systems of supervised machine learning in omics data analysis. The advantage of SVM compared to that of other algorithms is its good accuracy and use of fewer parameters to be optimized, even if the data dimension is large. However, the disadvantages of the SVM algorithm are the large calculation costs as the amount of training data increases; therefore, feature standardization will be needed.

\section{Identification of early detection biomarkers in NSCLC using omics data and AI}

\section{The application of Al in imaging diagnostics for NSCLC} screening

Most patients with NSCLC have advanced stage disease with distant metastasis at the initial diagnosis. The five-year survival rate of patients diagnosed at stage IV NSCLC is only $6.0 \%$ for patients that receive historic cytotoxic chemotherapy regimens, while the five-year survival rate dramatically rise to around $70-90 \%$ for patients diagnosed with stage I NSCLC [41]. Therefore, early detection of NSCLC is extremely effective toward improving the survival rate of patients. In 2011, the National Lung Screening Trial (NLST) showed that low-dose CT (LD-CT) screening for lung cancer reduced the relative mortality by $20 \%$ [42]. The all-cause mortality rate was $6.7 \%$ lower in the LD-CT group compared to that in the X-ray group. The US Preventive Services Task Force recommends an annual LD-CT screening test for high-risk populations, which comprises patients with a smoking history of at least 30 pack-years and an age of 55 to 80 years. However, the inclusion criteria excluded young subjects and never-smoker or light-smoker populations. Furthermore, LD-CT screening is costly with high false positive rates because of the detection of benign pulmonary nodules [43].

To detect ever-smaller lung tumors and to improve the accuracy of CT screening, the development of AIbased screening methods for all populations is rapidly progressing. Currently, complex algorithms and various types of software devices have been utilized to develop AI-based screening methods, and these are mainly categorized into two systems, namely, computer-aided detection (CADe) system and computer-aided diagnosis (CADx) system [44]. The CADe system, which highlights the detection of small nodules, has been engineered to improve radiologist sensitivity in identifying nodules. The CADx platforms can support diagnosis of pre-identified lesions when clinicians evaluate malignancy risk or conduct clinical decision-making. The development of both the systems is indeed important for improving diagnosis correctness, early diagnosis, and reducing diagnostic variation owing to clinician's subjectivity. However, most of the recent studies involve small sample sizes or no validated models, and therefore, AI-based screening methods are not enough for clinical application at this point of time.

To surmount the current difficulties, several frameworks of academia-industry collaboration have been gradually established worldwide. Optellum Ltd., a company that specializes in image analysis of lung cancer diagnosis, developed a machine leaning algorithm called the lung cancer prediction convolutional neural network (LCP-CNN), which was initially trained using the NLST data under guidance from experienced thoracic radiologists at Oxford University Hospitals [27, 45]. Subsequently, to compare the performance of LCP-CNN with that of the Brock University model, recommended by United Kingdom (UK) guidelines, Baldwin et al. conducted a validation study by retrospectively collecting data from 5-15 mm lung nodules, which consisted of 1187 patients with 1397 nodules from three hospitals in the UK [45]. In this study, the area under the curve (AUC) for LCP-CNN was $89.6 \%$ compared with $86.8 \%$ for the Brock model $(\mathrm{p} \leq 0.005)$, resulting in a better discrimination ability of LCP-CNN with over $99.5 \%$ sensitivity compared with that of the Brock model. As another model of academia-industry collaboration, Ardila et al. used the TensorFlow platform of Google Inc., to develop a deep-learning model trained using 42,290 CT scan images from 14,851 patients, which is able to determine the malignancy of lung nodules without the need for human intervention [46]. The AI-equipped system detected minute malignant lung nodules in 6716 test cases with an accuracy of $94 \%$. The model performed better than six radiologists who made the diagnosis in the absence of previous CT images [46]. The approach was undertaken in a collaboration between Google, Northwestern University, and other institutions, and is one of the systems moving toward clinical adoption. Both these studies showed successful academia-industry collaboration on radiomics and AI-based screening, which can 
make the detection of early lung cancer more precise and accessible for all the population.

\section{The application of omics data and Al for identification of early detection biomarkers}

For the purpose of supplementing the false positivity of LD-CT screening or earlier detection of lung cancer than that achieved using CT, a variety of new technologies have been investigated over the past decade for the discovery of biomarkers from various biomolecules. For example, because of dramatic advances in the accuracy of mass determination and characterization of target proteins, mass spectrometry (MS) has been developed to analyze a diverse range of proteins, lipids, and metabolites. Currently, it is possible to detect extremely small amounts of protein from tiny cancers using the advanced MS technology. In addition, Taguchi et al. performed proteomics analysis using blood samples obtained from various cancer mouse models and found levels of the $\mathrm{N}$-terminal pro-peptide of surfactant protein B (proSFTPB) are characteristically increased in the blood of mice with lung cancer [47]. Diagnostic blood tests, including for pro-SFTPB, may be able to identify people with lung cancer up to 2 years earlier with about twice the sensitivity of the current LD-CT criteria [48-50]. This indicates the combination of LD-CT screening and detection of protein-based biomarkers will be truly effective for the accurate and early detection of lung cancer.

The use of machine-learning approaches and the accumulation of omics data in recent years have led to more sensitive and accurate detection of biomarkers. For instance, Noreldeen et al. described a non-targeted lipidomic approach based on ultra-high-performance liquid chromatography coupled with quadrupole timeof-flight MS in combination with two machine learning approaches (genetic algorithm and binary logistic regression) to screen candidate discriminating lipids and to define a combinational lipid biomarker in serum samples for distinguishing female patients with NSCLC [51]. They showed that fatty acid (FA) (20:4), FA (22:0), and lysophosphatidylethanolamine (20:4) can serve as a combinational biomarker for distinguishing female patients with early-stage NSCLC from healthy controls with good sensitivity and specificity and the AUC reaching 0.99 . In a study using machine learning to parse omics data other than protein-based data, Best et al. analyzed RNA biomarker panels from platelet-derived RNA-sequencing libraries using particle-swarm optimization (PSO)enhanced algorithms [52]. Their results showed accurate tumor-educated blood platelets (TEP)-based detection of early-stage NSCLC (AUC, 0.89). Because the characteristics AI are from a completely different perspectives than that of earlier reports, the AI studies may lead to the elucidation of molecular biological mechanisms of lung cancer progression, as well as the identification of biomarkers for its early detection. It is expected that AI in the future will be able to integrate diagnostic imaging with new biomarkers using the comprehensive analysis of omics data.

\section{Development of immune checkpoint inhibitors (ICIs) treatment of NSCLC based on Al analysis of OMICS data}

Current issues in the standard treatment strategy of NSCLC and further development of immune therapy

Pivotal phase III clinical trials have led to the worldwide approval of ICIs, such as programmed cell death 1 (PD1)/programmed death-ligand 1 (PD-L1) inhibitors and cytotoxic T-lymphocyte-associated protein 4 (CTLA-4) inhibitors, especially for non-squamous NSCLC without sensitizing Epidermal Growth Factor Receptor (EGFR) mutation or anaplastic lymphoma kinase (ALK) fusion, and squamous cell lung carcinoma [53-58]. Multiple treatment regimens, including $\mathrm{PD}-1 / \mathrm{PD}-\mathrm{L} 1$ inhibitors with CTLA-4 inhibitors and PD-1/PD-L1 inhibitors with or without CTLA-4 plus platinum-based chemotherapy, are currently standard treatment options (Fig. 2). However, the optimal regimen for individual patients remains unclear as these new treatment regimens were compared to traditional platinum-based chemotherapy as the control-arm in all the phase III studies. Furthermore, comparative clinical trials of these new regimens have not been conducted and "round robin" clinical trials comparing these regimens may be unrealistic. However, novel immune therapeutic agents other than PD-1/PD-L1 inhibitors and CTLA-4 inhibitors are currently being investigated in several clinical trial settings [59], suggesting multiple combination therapies of immune targeting drugs may be approved before long as additional standard treatment options. Accordingly, the development of patient selection strategies for individualized immunotherapy is an important issue. In recent decades, comprehensive analyses of tumor specimens combined with detailed clinical information have been performed in various clinical trials [60]. Although these large profiling datasets have the potential to benefit the discovery of novel prediction methods of immune therapeutic activity for individual patients, translational and reverse translational research has not been adequately conducted. Under these circumstances, machine-learning approaches are some of the most promising technologies for identifying new biomarkers from various omics data that can be used to drive individualized immunotherapy.

The evaluation of PD-L1 expression and tumor mutation burden (TMB) using immunohistochemistry have been widely adopted as markers for ICI treatment [61, 


\section{Recommended treatment options according to oncogene driver status and PD-L1 expression in non-small-cell lung cancer}

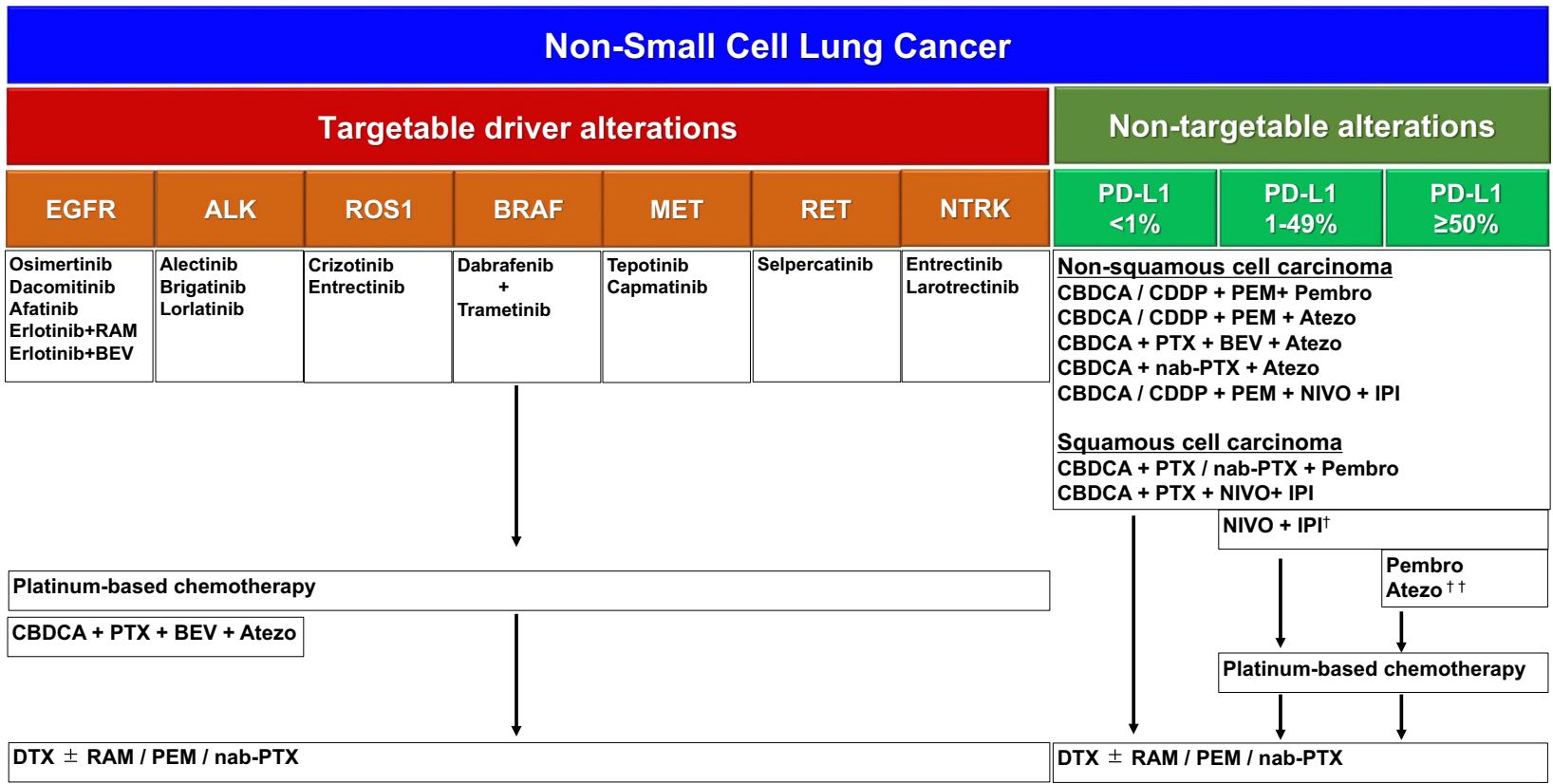

Fig. 2 Recommended treatment options according to oncogene driver status and PD-L1 expression in non-small-cell lung cancer. Next generation tyrosine kinase inhibitors for each driver oncogenic aberrations are approved one after another, and novel immune check point inhibitors combination with or without platinum-based chemotherapy are established as standard treatment options for non-small cell lung cancer without druggable alterations. ${ }^{\dagger}$ Nivolumab plus ipilimumab is an option for patients with PD-L1 tumor proportion score $<1 \%$ in addition to those with PD-L1 tumor proportion score $\geq 1 \%{ }^{\dagger+}$ High PD-L1 expression is defined as $\geq 50 \%$ of tumor cells or $\geq 10 \%$ of tumor-infiltrating immune cells by SP142 assay. CBDCA: Carboplatin; CDDP: Cisplatin; PTX: Paclitaxel; DTX: Docetaxel; PEM: Pemetrexed; nab-PTX: nanoparticle albumin bound Paclitaxel; BEV: bevacizumab; RAM: Ramucirumab; NIVO: Nivolumab; IPI: ipilimumab; Atezo: atezolizumab; Pembro: pembrolizumab

62]. However, the predictive ability is insufficient to adequately stratify patients for proper treatments compared to that of targeted oncogenic driver aberrations. These limitations are associated with immune responses being affected by tumor-cell specific features of NSCLC, immune-cell specific features, and the tumor microenvironment [63]. Using AI to establish a comprehensive prediction model for immunoblockade strategies will result in relevant advantages compared to that of traditional biomarker analysis. Once AI technology is able to identify populations with innate resistance to specific standard ICI regimens based on large clinical datasets of comprehensive tumor samples from clinical trials or real-world data (RWD), such populations will be a good target for further clinical trials of new ICI combination therapy aimed at overcoming innate resistance to ICI regimens. In addition to selecting suitable target populations for clinical trial settings, standard machine-learning approaches are expected to identify relevant biomarkers and clinical factors as each variable is interpretable using machine-learning methods. Furthermore, it will be possible to conduct subsequent reverse-translational research based on AI-driven interpretable biomarker profiling to determine the biological mechanism of primary resistance to specific standard ICI regimens. The harmonization between biological approaches and AI technology, supported by basic biological rationale, should foster the next generation of clinical trials with improved probability of positive clinical trial results.

Prognostic biomarker of ICl treatments using omics and AI Much of the accumulated evidence regarding the relationship between specific driver gene mutations and the immune microenvironment is based on recent NGS analyses. Two tumor suppressor genes in NSCLC, serine/ threonine kinase 11 (STK11) and Kelch-like ECH-associated protein 1 (KEAP1), are widely known as representative inactivated mutations with immunosuppressed phenotypes, regardless of PD-L1 expression and TMB [64-66].

Liver kinase B1 (LKB1), which is encoded by $S T K 11$, regulates cell polarity and functions as a tumor suppressor with germline mutations in this gene being related to the autosomal dominant disorder Peutz-Jeghers 
syndrome [67]. LKB1 inactivation is detected in approximately $20 \%$ of lung adenocarcinomas, effects tumor initiation, and uniquely confers invasive and metastatic properties through the reprograming of energy metabolism, such as glucose/FA uptake and pyrimidine/ purine balance [68-71]. In the NSCLC tumor microenvironment, LKB1 inactivation is shown to downregulate PD-L1 expression and promote proinflammatory cytokine production to suppress T-cell infiltration [72].

Meanwhile, KEAP1 is an adaptor for a cullin-3 (CUL3)based ubiquitin ligase and is involved in the control of oxidative stress to facilitate ubiquitination and the subsequent proteolysis of nuclear factor erythroid 2-related factor 2 (NRF2), which is a master regulator of the antioxidant response. Loss of KEAP1 or CUL3 function results in constant NRF2 activation and the tumors exhibit resistance to radiotherapy and cytotoxic chemotherapy [73-75]. NSCLC with LKB1 inactivation and/ or disruption of the NRF2-KEAP1-CUL3 complex are widely known to demonstrate an aggressive clinical course, shorter survival rates, and resistance to ICIs treatments. Furthermore, recent multi-omics analysis has determined that activating mutations in receptor tyrosine kinases genes, such as EGFR mutations, human epidermal growth factor receptor 2 (HER2) point mutations and amplifications, MET Proto-Oncogene, Receptor Tyrosine Kinase (MET) amplification, fibroblast growth factor receptor 1 (FGFR1) amplification, and insulin like growth factor 1 receptor (IGF1R) amplification, are linked to primary resistance to ICIs, independent of PD-L1 expression and TMB [76]. Among these, EGFR activation shows various immunosuppressive mechanisms to suppress tumor-infiltrating lymphocytes, including the expression of CD73 and secretion of T-cell inhibitory molecules [77-80]. Conversely, several driver gene mutations, including AT-rich interactive domain-containing protein $1 A$ (ARID1A), Janus kinase 1 (JAK1), and Janus kinase 2 (JAK2) mutations and co-occurring KRAS mutations and TP53 inactivation, are associated with T-cell infiltration and reflect favorable responses to ICIs therapies with high expression of tumor antigens $[66,76,81$, 82]. Therefore, the widespread utilization of NGS-based testing, which is currently tending to decline in cost, will help guide the selection of good responders to ICIs.

Other monolayer omics analyses have also led to the elucidation of the immune-microenvironment of individual tumors and to the establishment of predictors for ICIs therapeutic efficacy. For instance, examination of whole-exome signatures of mutagenic biological processes within tumor specimens has found an enrichment of the $\mathrm{C}>\mathrm{A}$ transversion-rich molecular tobacco-smoking signature in patients with durable benefits by ICIs treatment [83]. When a tobacco-smoking signature is detected, the total number of single-base substitutions is shown to associate with TMB and more accurately predicts ICIs response than TMB [76]. Tumor-specific neopeptides linked to T-cell infiltrates in tumors and the clinical efficacy of ICIs have also been well investigated in various cancer types $[84,85]$. For effective tumor killing, $\mathrm{CD} 8+\mathrm{T}$ cells must recognize the neo-peptides presented by human leukocyte antigen class I (HLA-I) molecules. Deficiency of antigen presentation is associated with immune escape through both HLA class I germline homozygosity and the loss of heterozygosity, which then influences the response of cancer to ICIs [86, 87]. However, these monolayer omics analyses may be less effective in accurately predicting the outcome of treatment with ICIs and multimodal approaches might be needed [76].

In an effort to accurately classify patients with ICI response, Lu et al. attempted to establish a proper model using machine learning and whole-exome sequencing data [37]. They used metastatic melanoma as training data and validation was conducted using a NSCLC dataset. From the initial model, which considered 2139 mutations, their machine learning technique selected 161 mutations (11\%). In the NSCLC cohort, the high-weightTMB group was found to be associated with better survival and better 6-month clinical benefit was predicted (AUC $=0.83$ ). Interestingly, among the 161 mutations, only nine genes $(<6 \%)$ had negative coefficients and the weighted gene mutation selected by their machine-learning technique was consistent with previous mutation load markers based on molecular omics analysis.

Meanwhile, Wiesweg et al. conducted machine learning approaches on RNA expression of a 770-gene panel covering immune-related genes in patients with advanced NSCLC, in combination with PD-L1 immunohistochemistry [39]. The model prediction plus PD-L1 positivity identified NSCLC patients with highly favorable outcomes.

In addition to NGS analysis, integrated analysis based on multi-omics data including tumor-adjacent tissue, should allow for construction of new models for the accurate prediction of therapeutic efficacy. In addition to the application of machine learning for omics data analysis, several studies have developed deep learning to predict ICIs efficacies using pathological images and clinical information. For instance, Khalid et al. conducted integrative analysis of spatial histological images by training deep-learning algorithms in addition to analysis of multi-region exome and RNA-sequencing data in 100 patients with NSCLC [88]. The study demonstrated that lung adenocarcinomas with more than one immune-cold region were at significantly higher risk of cancer relapse, regardless of the number of total regions sampled and the 
immune phenotypes of the other regions. In this way, AIbased analysis using omics data and clinical information can provide a completely new perspective on predicting therapeutic effects. As the next research strategy, integration of multilayer omics data with machine-learning analysis in combination with analysis of clinical information, such as CT and/or histopathological images, by training deep-learning will provide currently insensible prediction models.

\section{Future direction and challenges of using Al in NSCLC with druggable mutations}

\section{Current issues of molecular targeted drug discovery} and clinical trials in NSCLC with oncogenic driver aberrations

Several oncogenic driver mutations and oncogenic fusions have been established as therapeutic targets for NSCLC. In such oncogenic driver aberrations of NSCLC, EGFR, ALK, MET, and B-Raf proto-oncogene serine/threonine kinase (BRAF) mutations, and $A L K$, ROS proto-oncogene 1 receptor tyrosine kinase (ROS1), ret proto-oncogene (RET), and neurotrophic receptor tyrosine kinase (NTRK) fusions have been identified and the clinical benefit of several tyrosine kinase inhibitors (TKI) targeting these oncogenic driver mutations and fusions have been proven by well-designed clinical trials (Fig. 2) [41]. Targeted therapy of oncogenic driver mutations and oncogenic fusions in NSCLC achieve higher response rates with longer duration of progression free survival (PFS) compared to conventional cytotoxic agents. However, several issues remain in the further development of individualized treatment strategies for oncogenic driver mutations and fusions. For instance, the clinical benefit for each oncogenic driver aberrations depends on both the inhibitory ability of a specific targeted oncogenic driver aberration and tolerability. Thus, the discovery of new compounds that exhibit highly selective inhibitory effect for targeted oncogenic driver aberrations is one of the most crucial steps in the development of new standard treatments.

As an example, we review the history of developing an $A L K$-fusion targeted therapy. The discovery of ALK dates back to 1994 when a chromosomal rearrangement, $\mathrm{t}(2 ; 5)$, resulting in a nucleophosmin (NPM1)-ALK fusion was described in anaplastic large-cell lymphoma [89]. More than a decade later, subsequent work identified the $A L K$ fusion proteins as oncogenic driver alterations in a variety of cancer types. Among them, the echinoderm microtubule-associated protein-like 4 (EML4)-ALK fusion was recognized in 2007 as a representative oncogenic driver fusion in approximately 3-7\% of NSCLC [90]. Several years later, the first approved agent for $A L K$ fusion, crizotinib, was shown to exhibit superiority over cytotoxic chemotherapy [91, 92]. However, because of its inhibitory activity on several tyrosine kinases in addition to ALK, such as ROS1 and MET among others, crizotinib frequently causes various adverse events (AEs), including nausea, bradycardia and transient visual disorders [93]. The severe AEs sometimes lead to the targeted therapy being discontinued. Therefore, the development of a new agent with high selectivity for oncogenic $A L K$-fusion signaling was necessary as a next step to achieve further long-term tumor control with less toxicity. The second generation ALK-TKI alectinib was designed to inhibit the ALK tyrosine kinase with high selectivity [94]. Based on the results of three phase III clinical trials that proved the superiority of alectinib with PFS as the primary endpoint over that of crizotinib with less toxicity, alectinib was approved in 2017 as a first-line standard agent [9597]. This developmental history of a molecular targeted therapy is one of the success stories for the patients with oncogenic driver aberrations; however, drug discovery and developments starting from traditional screening methods to clinical trials is an extremely expensive and time-consuming procedure. Moreover, as another major issue, less than $10 \%$ of agents entering clinical trial settings achieve successful results and the Food and Drug Administration approval [98]. Furthermore, approximately $20 \%$ of tumors show innate resistance and early tumor progression based on several biological characteristics, such as intratumoral heterogeneity and other driver mutations. The remaining tumors subsequently acquire resistance through various molecular mechanisms, including secondary mutation of the same driver gene or activation of other oncogenic signals. Most oncogenic driver aberrations are themselves only a relatively rare fraction of the tumor cell population. The subpopulation classified by a specific resistant mechanism of each oncogenic driver aberrations is increased through the variety of aberrations [99]. Therefore, screening promising new targeted strategies for overcoming resistance mechanisms determined by oncogenic aberrations in each specific subpopulation and conducting multiple phase I/II trials based on traditional methods seems unrealistic.

\section{Potential role of $\mathrm{Al}$ in development of new treatment strategies targeting oncogenic driver aberrations}

The discovery of highly selective inhibitors that target oncogenic driver aberrations is a crucial step in the ultimate approval of a novel standard molecular targeted therapy. AI is expected to play several roles in the development of new treatment strategies (Fig. 3). First, $\mathrm{AI}$ enables the virtual screening of targeted lead compounds using multiple public databases, such as TCGA, the Human Protein Atlas and DrugBank, and PubChem. 


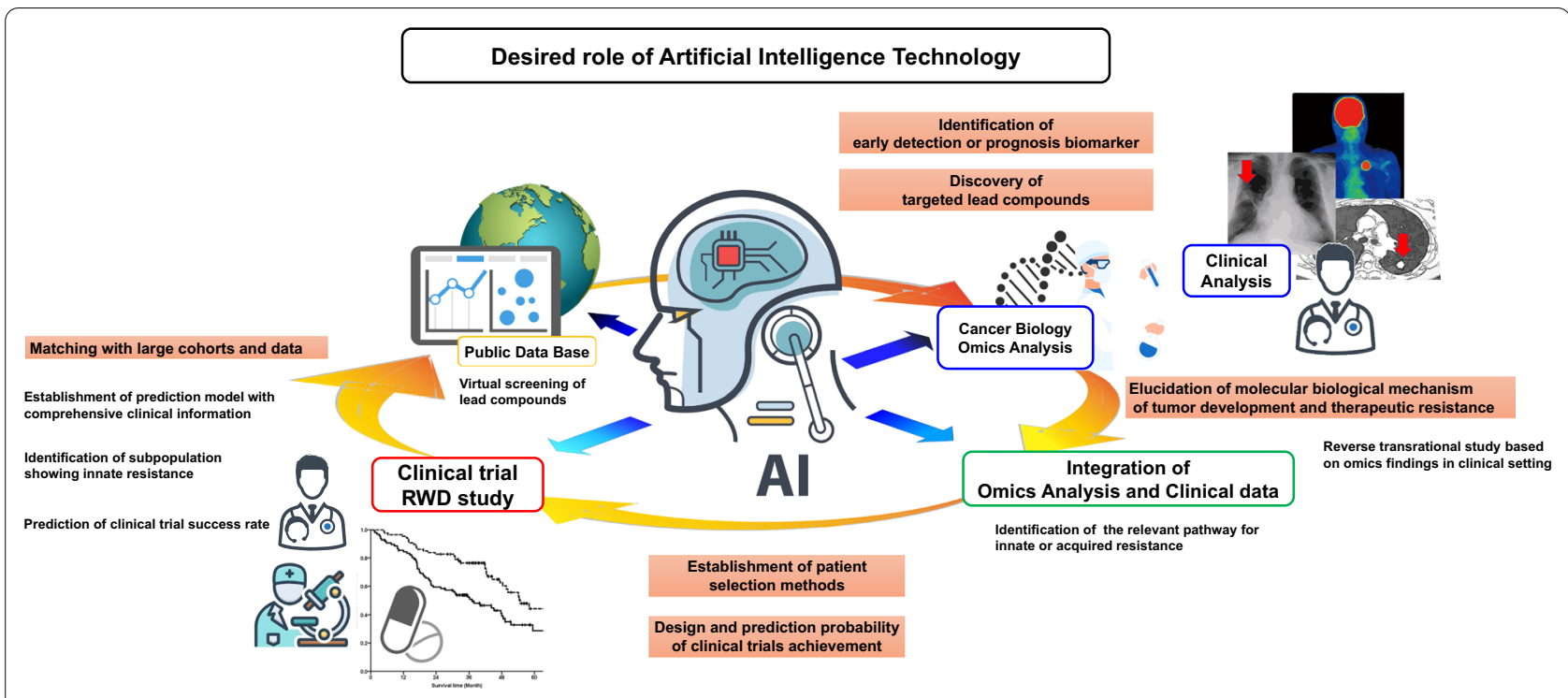

Fig. 3 Future direction and potential role of artificial intelligence for development of new treatment strategies

AI-based virtual screening supports the identification of candidate compounds with highly specific selectivity for targeted oncogenic driver aberrations and low toxicity. For example, Istvan et al. reported an AI-assisted computational method, which is a proprietary technology of Oncompass Medicine Inc., to prioritize potential molecular targeted therapies based on the complex individual molecular profile of the tumor in each patient [100]. They analyzed the clinical benefits of the digital drugassignment system using the data from the SHIVA01 precision oncology clinical trial, and showed that the system identified substantial molecular targets with the fitting inhibitors, including in lung cancer patients, such as FMS Related Receptor Tyrosine Kinase 3 mutation with sorafenib and Androgen receptor expression with abiraterone. These findings indicate that the AI-assisted computational systems for prioritization of potential molecular targeted therapies would be promising to improve the clinical benefits of precision oncology. As another example, in recent studies, it has been reported that the discovery of selective heat shock protein 90 inhibitors and an aurora A inhibitor was driven by virtual screening $[101,102]$. These ligand-based virtual screening methods will be a powerful tool in selecting a new and ideal inhibitor against previously identified molecular targets. Because resistance to targeted drugs of oncogenic driver aberrations can emerge through secondary oncogenic driver aberrations that exhibit various molecular mechanism of resistance, the virtual screening of compounds can promote the cost-effective development of treatment strategies for overcoming heterogeneous mechanism of resistance. Traditional screening methods combined with conducting multiple phase I/II trials are currently required to replenish the pool of potential innovative development strategies and new drugs for targeting oncogenic driver aberrations. In addition to the discovery of targeted compounds, AI will be contributing on the prediction of success rates of clinical trials. Indeed, Gayvert et al. reported that a new data-driven approach is able to predict clinical toxicity and may identify compounds in clinical trials with acceptable toxicity [102]. Improving the probability of success for clinical trials based on AI would also help resolve the current issue of a limited availability of patients with rare oncogenic driver aberrations.

\section{Prospects}

Precision medicine in the treatment of lung cancer has shown a dramatic growth with progression in harmonization of molecular cancer biology and AI-based technology. AI, radiomics, and molecular cancer biology exhibit mutual influence, and can generate powerful AI systems for further development of individual treatment strategies. Although accumulating recent omics data have consecutively provided a variety of new biological insights, the analysis may have been beyond the capabilities of manual analysis. Therefore, establishing new framework for analyzing huge size of omics data, such as academia-industry collaboration and academia-government technological collaboration, will be important as well as the AI development. With regard to radiology and molecular targeted therapies, some academia-industry collaborations have successfully complemented each other $[45,46,100]$, and AI-based 
screening has been accelerated toward clinical applications. Prospectively, these frameworks, which can lead to further progression of inter-industry activities, and medical AI systems could be a detector of microchanges in patients that can go unnoticed by human eyes, and be a selector of suitable treatments for individual patients to support clinicians, resulting in more early intervention and in improving the quality of life of patients.

Moreover, comprehensive profiles of individual omics data are increasingly important not only to patients but also to their families and blood relatives. Additionally, recent AI-based systems for multi-omics analyses have an increased possibility of accidental and unexpected discoveries to affect an individual's life. Patients should have the opportunity to know how their data is being shared and used, and the enormous individual data should be protected against risks of disclosure. Therefore, omics information holders have ethical and legal obligations, big responsibilities for data stewardship, and regulatory issues for decision-making. Under the current law structure, these ethical and legal issues may not be satisfactorily served with regard to various aspects including those in the area of intellectual property. In parallel with the rapid development of AI-based omics data analysis, revisions to the legal framework would also be needed.

\section{Conclusions}

Machine-learning and deep-learning technologies have undergone relevant advances, enabling the analysis large omics datasets and clinical information. Toward improving the prognosis of patients with NSCLC, AI has shown breakthroughs in potentially resolving current issues in the development of new treatment strategies, including for ICIs and molecular targeted therapy. These include, (1) identification of early detection or prognosis biomarkers, (2) elucidation of molecular biological mechanisms of tumor development and therapeutic resistance, (3) establishment of patient selection and stratification methods, (4) discovery of lead targeted compounds, and (5) design of clinical trials and prediction of their probable achievements or outcomes. In the coming decade, researchers will need to select suitable AI algorithms for analyzing expansive amounts of omics data and clinical information. Harmonization of molecular cancer biology and AI technology will dramatically improve research strategies and accelerate the creation of efficient outcomes that are beyond simply human capability.

\section{Acknowledgements}

The authors would like to thank Enago (www.enago.jp) for the English language review.
Authors' contributions

IT, TF and MM participated in original draft preparation, collection and analysis of the data, conceptualization, review, proofreading and editing of the manuscript. All authors read and approved the final manuscript.

\section{Funding}

This research was supported by a grant awarded to I. Tanaka from Grant-in-Aid for Scientific Research (B) $20 \mathrm{H} 03689$ of the Japan Society for the Promotion of Science.

\section{Availability of data and materials \\ Yes.}

\section{Declarations}

Ethics approval and consent to participate

Not applicable.

\section{Consent for publication}

Not applicable.

\section{Competing interests}

The authors declare that they have no competing interests.

\section{Author details}

${ }^{1}$ Department of Respiratory Medicine, Nagoya University Graduate School of Medicine, 65 Tsurumai-cho, Showa-ku, Nagoya 466-8550, Japan. ${ }^{2}$ Center for Healthcare Information Technology (C-HiT), Nagoya University, Nagoya, Japan.

Received: 1 June 2021 Accepted: 19 Auqust 2021

Published online: 26 August 2021

References

1. Waldron D. Cancer genomics: a multi-layer omics approach to cancer. Nat Rev Genet. 2016;17(8):436-7.

2. Li J, Chen $H$, Wang $Y$, Chen MM, Liang $H$. Next-generation analytics for omics data. Cancer Cell. 2021:39(1):3-6.

3. Hoadley KA, Yau C, Hinoue T, Wolf DM, Lazar AJ, Drill E, Shen R, Taylor AM, Cherniack AD, Thorsson V, et al. Cell-of-origin patterns dominate the molecular classification of 10,000 tumors from 33 types of cancer. Cell. 2018;173(2):291-304.

4. Bailey MH, Tokheim C, Porta-Pardo E, Sengupta S, Bertrand D, Weerasinghe A, Colaprico A, Wendl MC, Kim J, Reardon B, et al. Comprehensive characterization of cancer driver genes and mutations. Cell. 2018;174(4):1034-5.

5. Sanchez-Vega F, Mina M, Armenia J, Chatila WK, Luna A, La KC, Dimitriadoy S, Liu DL, Kantheti HS, Saghafinia S, et al. Oncogenic signaling pathways in the cancer genome atlas. Cell. 2018;173(2):321-37.

6. Consortium ITP-CAoWG. Pan-cancer analysis of whole genomes. Nature. 2020;578(7793):82-93.

7. Gerstung M, Jolly C, Leshchiner I, Dentro SC, Gonzalez S, Rosebrock D, Mitchell TJ, Rubanova Y, Anur P, Yu K, et al. The evolutionary history of 2,658 cancers. Nature. 2020;578(7793):122-8.

8. Reiter JG, Baretti M, Gerold JM, Makohon-Moore AP, Daud A, lacobuzioDonahue CA, Azad NS, Kinzler KW, Nowak MA, Vogelstein B. An analysis of genetic heterogeneity in untreated cancers. Nat Rev Cancer. 2019;19(11):639-50.

9. Paczkowska M, Barenboim J, Sintupisut N, Fox NS, Zhu H, Abd-Rabbo D, Mee MW, Boutros PC, Drivers P, Functional Interpretation Working, et al. Integrative pathway enrichment analysis of multivariate omics data. Nat Commun. 2020;11(1):735.

10. Reyna MA, Haan D, Paczkowska M, Verbeke LPC, Vazquez M, Kahraman A, Pulido-Tamayo S, Barenboim J, Wadi L, Dhingra P, et al. Pathway and network analysis of more than 2500 whole cancer genomes. Nat Commun. 2020:11(1):729.

11. Escala-Garcia M, Abraham J, Andrulis IL, Anton-Culver H, Arndt V, Ashworth A, Auer PL, Auvinen P, Beckmann MW, Beesley J, et al. A network 
analysis to identify mediators of germline-driven differences in breast cancer prognosis. Nat Commun. 2020;11(1):312.

12. Kuenzi BM, Ideker T. A census of pathway maps in cancer systems biology. Nat Rev Cancer. 2020;20(4):233-46.

13. Baslan T, Hicks J. Unravelling biology and shifting paradigms in cancer with single-cell sequencing. Nat Rev Cancer. 2017;17(9):557-69.

14. Dawes TJW, de Marvao A, Shi W, Fletcher T, Watson GMJ, Wharton J, Rhodes CJ, Howard L, Gibbs JSR, Rueckert D, et al. Machine learning of three-dimensional right ventricular motion enables outcome prediction in pulmonary hypertension: a cardiac MR imaging study. Radiology. 2017;283(2):381-90.

15. Hosny A, Parmar C, Coroller TP, Grossmann P, Zeleznik R, Kumar A, Bussink J, Gillies RJ, Mak RH, Aerts H. Deep learning for lung cancer prognostication: a retrospective multi-cohort radiomics study. PLoS Med. 2018;15(11):e1002711.

16. Hosny A, Parmar C, Quackenbush J, Schwartz LH, Aerts H. Artificial intelligence in radiology. Nat Rev Cancer. 2018;18(8):500-10.

17. Yamamoto Y, Tsuzuki T, Akatsuka J, Ueki M, Morikawa H, Numata Y, Takahara T, Tsuyuki T, Tsutsumi K, Nakazawa R, et al. Automated acquisition of explainable knowledge from unannotated histopathology images. Nat Commun. 2019;10(1):1-9.

18. Zhang Z, Chen P, McGough M, Xing F, Wang C, Bui M, Xie Y, Sapkota M, Cui L, Dhillon J, et al. Pathologist-level interpretable whole-slide cancer diagnosis with deep learning. Nat Mach Intell. 2019;1 (5):236-45.

19. Wentzensen N, Lahrmann B, Clarke MA, Kinney W, Tokugawa D, Poitras N, Locke A, Bartels L, Krauthoff A, Walker J, et al. Accuracy and efficiency of deep-learning-based automation of dual stain cytology in cervical cancer screening. J Natl Cancer Inst. 2021;113(1):72-9.

20. Biswas N, Chakrabarti S. Artificial intelligence (Al)-based systems biology approaches in multi-omics data analysis of cancer. Front Oncol. 2020;10(2224):588221.

21. Siegel RL, Miller KD, Jemal A. Cancer statistics, 2020. CA Cancer J Clin. 2020;70(1):7-30.

22. Hanna N, Johnson D, Temin S, Baker S Jr, Brahmer J, Ellis PM, Giaccone G, Hesketh PJ, Jaiyesimi I, Leighl NB, et al. Systemic therapy for stage IV non-small-cell lung cancer: American Society of Clinical Oncology Clinical Practice Guideline Update. J Clin Oncol. 2017;35(30):3484-515.

23. Akamatsu H, Ninomiya K, Kenmotsu H, Morise M, Daga H, Goto Y, Kozuki T, Miura S, Sasaki T, Tamiya A, et al. The Japanese Lung Cancer Society Guideline for non-small cell lung cancer, stage IV. Int J Clin Oncol. 2019;24(7):731-70

24. Paz-Ares L Ciuleanu TE, Cobo M, Schenker M, Zurawski B, Menezes J, Richardet E, Bennouna J, Felip E, Juan-Vidal O, et al. First-line nivolumab plus ipilimumab combined with two cycles of chemotherapy in patients with non-small-cell lung cancer (CheckMate 9LA): an international, randomised, open-label, phase 3 trial. Lancet Oncol. 2021;22(2):198-211

25. Hellmann MD, Paz-Ares L, Bernabe Caro R, Zurawski B, Kim SW, Carcereny Costa E, Park K, Alexandru A, Lupinacci L, de la Mora JE, et al. Nivolumab plus Ipilimumab in advanced non-small-cell lung cancer. $N$ Engl J Med. 2019;381(21):2020-31.

26. Rajkomar A, Dean J, Kohane I. Machine Learning in Medicine. N Engl J Med. 2019:380(14):1347-58.

27. Lecun Y, Bengio Y, Hinton G. Deep learning. Nature. 2015:521(7553):436-44

28. Coudray N, Ocampo PS, Sakellaropoulos T, Narula N, Snuderl M, Fenyo D, Moreira AL, Razavian N, Tsirigos A. Classification and mutation prediction from non-small cell lung cancer histopathology images using deep learning. Nat Med. 2018;24(10):1559-67.

29. Ehteshami Bejnordi $B$, Veta $M$, Johannes van Diest $P$, van Ginneken $B$, Karssemeijer N, Litjens G, van der Laak J, the CAMELYON16 Consortium, Hermsen M, Manson QF et al. Diagnostic assessment of deep learning algorithms for detection of lymph node metastases in women with breast cancer. JAMA 2017, 318(22):2199-2210.

30. Zhang ZZ, Chen PJ, McGough M, Xing FY, Wang CB, Bui M, Xie YP, Sapkota M, Cui L, Dhillon J, et al. Pathologist-level interpretable whole-slide cancer diagnosis with deep learning. Nat Mach Intelli. 2019;1 (5):236.

31. Lu MT, Raghu VK, Mayrhofer T, Aerts H, Hoffmann U. Deep learning using chest radiographs to identify high-risk smokers for lung cancer screening computed tomography: development and validation of a prediction model. Ann Intern Med. 2020;173(9):704-13.
32. Yamamoto Y, Tsuzuki T, Akatsuka J, Ueki M, Morikawa H, Numata Y, Takahara T, Tsuyuki T, Tsutsumi K, Nakazawa R, et al. Automated acquisition of explainable knowledge from unannotated histopathology images. Nat Commun. 2019;10(1):5642.

33. Park SH, Han K. Methodologic guide for evaluating clinical performance and effect of artificial intelligence technology for medical diagnosis and prediction. Radiology. 2018:286(3):800-9.

34. Lakhani P, Sundaram B. Deep learning at chest radiography: automated classification of pulmonary tuberculosis by using convolutional neural networks. Radiology. 2017;284(2):574-82.

35. Lakshmanaprabu SK, Mohanty SN, Shankar K, Arunkumar N, Ramirez G. Optimal deep learning model for classification of lung cancer on CT images. Future Gener Comput Syst. 2019;92:374-82.

36. Elia G, Ferrari SM, Galdiero MR, Ragusa F, Paparo SR, Ruffilli I, Varricchi G, Fallahi P, Antonelli A. New insight in endocrine-related adverse events associated to immune checkpoint blockade. Best Pract Res Clin Endocrinol Metab. 2019;34:101370.

37. Lu M, Wu KH, Trudeau S, Jiang M, Zhao J, Fan E. A genomic signature for accurate classification and prediction of clinical outcomes in cancer patients treated with immune checkpoint blockade immunotherapy. Sci Rep. 2020;10(1):20575.

38. Best MG, In't Veld S, Sol N, Wurdinger T. RNA sequencing and swarm intelligence-enhanced classification algorithm development for bloodbased disease diagnostics using spliced blood platelet RNA. Nat Protoc. 2019;14(4):1206-34

39. Wiesweg M, Mairinger F, Reis H, Goetz M, Kollmeier J, Misch D, StephanFalkenau S, Mairinger T, Walter RFH, Hager T, et al. Machine learning reveals a PD-L1-independent prediction of response to immunotherapy of non-small cell lung cancer by gene expression context. Eur J Cancer. 2020;140:76-85.

40. Guan S, Jia B, Chao K, Zhu X, Tang J, Li M, Wu L, Xing L, Liu K, Zhang $L$, et al. UPLC-QTOF-MS-based plasma lipidomic profiling reveals biomarkers for inflammatory Bowel disease diagnosis. J Proteome Res. 2020;19(2):600-9.

41. Ettinger DS, Wood DE, Aggarwal C, Aisner DL, Akerley W, Bauman JR, Bharat A, Bruno DS, Chang JY, Chirieac LR, et al. NCCN guidelines insights: non-small cell lung cancer, version 1.2020. J Natl Compr Canc Netw. 2019:17(12):1464-72.

42. National Lung Screening Trial Research, Aberle DR, Adams AM, Berg CD, Black WC, Clapp JD, Fagerstrom RM, Gareen IF, Gatsonis C, Marcus PM, et al. Reduced lung-cancer mortality with low-dose computed tomographic screening. N Engl J Med. 2011:365(5):395-409.

43. Goulart BH, Bensink ME, Mummy DG, Ramsey SD. Lung cancer screening with low-dose computed tomography: costs, national expenditures, and cost-effectiveness. J Natl Compr Canc Netw. 2012;10(2):267-75.

44. Firmino M, Angelo G, Morais H, Dantas MR, Valentim R. Computer-aided detection (CADe) and diagnosis (CADx) system for lung cancer with likelihood of malignancy. Biomed Eng Online. 2016;15:2.

45. Baldwin DR, Gustafson J, Pickup L, Arteta C, Novotny P, Declerck J, Kadir T, Figueiras C, Sterba A, Exell A, et al. External validation of a convolutional neural network artificial intelligence tool to predict malignancy in pulmonary nodules. Thorax. 2020;75(4):306-12.

46. Ardila D, Kiraly AP, Bharadwaj S, Choi B, Reicher JJ, Peng L, Tse D, Etemadi M, Ye W, Corrado G, et al. End-to-end lung cancer screening with three-dimensional deep learning on low-dose chest computed tomography. Nat Med. 2019;25(6):954-61.

47. Taguchi A, Politi K, Pitteri SJ, Lockwood WW, Faca VM, Kelly-Spratt K, Wong $\mathrm{CH}$, Zhang Q, Chin A, Park KS, et al. Lung cancer signatures in plasma based on proteome profiling of mouse tumor models. Cancer Cell. 2011;20(3):289-99

48. Sin DD, Tammemagi CM, Lam S, Barnett MJ, Duan X, Tam A, Auman H, Feng Z, Goodman GE, Hanash S, et al. Pro-surfactant protein B as a biomarker for lung cancer prediction. J Clin Oncol. 2013;31(36):4536-43.

49. Taguchi A, Hanash S, Rundle A, McKeague IW, Tang D, Darakjy S, Gaziano JM, Sesso HD, Perera F. Circulating pro-surfactant protein B as a risk biomarker for lung cancer. Cancer Epidemiol Biomarkers Prev. 2013;22(10):1756-61.

50. Integrative Analysis of Lung Cancer E, Risk Consortium for Early Detection of Lung, Guida F, Sun N, Bantis LE, Muller DC, Li P, Taguchi A, Dhillon D, Kundnani DL, et al. Assessment of lung cancer risk on 
the basis of a biomarker panel of circulating proteins. JAMA Oncol. 2018;4(10):e182078

51. Noreldeen HAA, Du L, Li W, Liu X, Wang Y, Xu G. Serum lipidomic biomarkers for non-small cell lung cancer in nonsmoking female patients. J Pharm Biomed Anal. 2020;185:113220.

52. Best MG, Sol N, In 't Veld S, Vancura A, Muller M, Niemeijer AN, Fejes AV, Tjon Kon Fat LA, Huis In 't Veld AE, Leurs C, et al. Swarm intelligenceenhanced detection of non-small-cell lung cancer using tumor-educated platelets. Cancer Cell. 2017;32(2):238-25.

53. Gandhi L, Rodriguez-Abreu D, Gadgeel S, Esteban E, Felip E, De Angelis F, Domine M, Clingan P, Hochmair MJ, Powell SF, et al. Pembrolizumab plus chemotherapy in metastatic non-small-cell lung cancer. N Engl J Med. 2018;378(22):2078-92.

54. Reck M, Rodriguez-Abreu D, Robinson AG, Hui R, Csoszi T, Fulop A, Gottfried M, Peled N, Tafreshi A, Cuffe S, et al. Pembrolizumab versus chemotherapy for PD-L1-positive non-small-cell lung cancer. N Engl J Med. 2016;375(19):1823-33.

55. MokTSK, Wu YL, Kudaba I, Kowalski DM, Cho BC, Turna HZ, Castro G $\mathrm{Jr}$, Srimuninnimit V, Laktionov KK, Bondarenko I, et al. Pembrolizumab versus chemotherapy for previously untreated, PD-L1-expressing, locally advanced or metastatic non-small-cell lung cancer (KEYNOTE-042): a randomised, open-label, controlled, phase 3 trial. Lancet. 2019;393(10183):1819-30.

56. Paz-Ares L, Luft A, Vicente D, Tafreshi A, Gumus M, Mazieres J, Hermes B, Cay Senler F, Csoszi T, Fulop A, et al. Pembrolizumab plus chemotherapy for squamous non-small-cell lung cancer. N Engl J Med. 2018;379(21):2040-51.

57. Socinski MA, Jotte RM, Cappuzzo F, Orlandi F, Stroyakovskiy D, Nogami N, Rodriguez-Abreu D, Moro-Sibilot D, Thomas CA, Barlesi F, et al. Atezolizumab for first-line treatment of metastatic nonsquamous NSCLC. N Engl J Med. 2018;378(24):2288-301.

58. Herbst RS, Giaccone G, de Marinis F, Reinmuth N, Vergnenegre A, Barrios $\mathrm{CH}$, Morise M, Felip E, Andric Z, Geater S, et al. Atezolizumab for firstline treatment of PD-L1-selected patients with NSCLC. N Engl J Med. 2020;383(14):1328-39.

59. Rocco D, Gregorc V, Della Gravara L, Lazzari C, Palazzolo G, Gridelli C. New immunotherapeutic drugs in advanced non-small cell lung cancer (NSCLC): from preclinical to phase I clinical trials. Expert Opin Investig Drugs. 2020;29(9):1005-23.

60. Safa H, Tamil M, Spiess PE, Manley B, Pow-Sang J, Gillbert SM, Safa F, Gonzalez BD, Oswald LB, Semaan A, et al. Patient-reported outcomes in clinical trials leading to cancer immunotherapy drug approvals from 2011 to 2018: a systematic review. J Natl Cancer Inst. 2021;113(5):532-42.

61. Lantuejoul S, Sound-Tsao M, Cooper WA, Girard N, Hirsch FR, Roden AC, Lopez-Rios F, Jain D, Chou TY, Motoi N, et al. PD-L1 testing for lung cancer in 2019: perspective from the IASLC Pathology Committee. J Thorac Oncol. 2020;15(4):499-519.

62. Hendriks LE, Rouleau E, Besse B. Clinical utility of tumor mutational burden in patients with non-small cell lung cancer treated with immunotherapy. Transl Lung Cancer Res. 2018;7(6):647-60.

63. Hiam-Galvez KJ, Allen BM, Spitzer MH. Systemic immunity in cancer. Nat Rev Cancer. 2021;21(6):345-59.

64. Skoulidis F, Heymach JV. Co-occurring genomic alterations in non-small-cell lung cancer biology and therapy. Nat Rev Cancer. 2019;19(9):495-509.

65. Skoulidis F, Goldberg ME, Greenawalt DM, Hellmann MD, Awad MM, Gainor JF, Schrock AB, Hartmaier RJ, Trabucco SE, Gay L, et al. STK11/ LKB1 mutations and PD-1 inhibitor resistance in KRAS-mutant lung adenocarcinoma. Cancer Discov. 2018;8(7):822-35.

66. Arbour KC, Jordan E, Kim HR, Dienstag J, Yu HA, Sanchez-Vega F, Lito P, Berger M, Solit DB, Hellmann M, et al. Effects of co-occurring genomic alterations on outcomes in patients with KRAS-mutant non-small cell lung cancer. Clin Cancer Res. 2018;24(2):334-40.

67. Hearle N, Schumacher V, Menko FH, Olschwang S, Boardman LA, Gille JJ, Keller JJ, Westerman AM, Scott RJ, Lim W, et al. Frequency and spectrum of cancers in the Peutz-Jeghers syndrome. Clin Cancer Res. 2006:12(10):3209-15.

68. Shackelford DB, Shaw RJ. The LKB1-AMPK pathway: metabolism and growth control in tumour suppression. Nat Rev Cancer. 2009;9(8):563-75.
69. Ji H, Ramsey MR, Hayes DN, Fan C, McNamara K, Kozlowski P, Torrice C, Wu MC, Shimamura T, Perera SA, et al. LKB1 modulates lung cancer differentiation and metastasis. Nature. 2007:448(7155):807-10.

70. Celiktas M, Tanaka I, Tripathi SC, Fahrmann JF, Aguilar-Bonavides C, Villalobos P, Delgado O, Dhillon D, Dennison JB, Ostrin EJ, et al. Role of CPS1 in cell growth, metabolism and prognosis in LKB1-inactivated lung adenocarcinoma. J Natl Cancer Inst. 2017;109(3):1-9.

71. Kim J, Hu Z, Cai L, Li K, Choi E, Faubert B, Bezwada D, RodriguezCanales J, Villalobos P, Lin YF, et al. CPS1 maintains pyrimidine pools and DNA synthesis in KRAS/LKB1-mutant lung cancer cells. Nature. 2017;546(7656):168-72.

72. Koyama S, Akbay EA, Li YY, Aref AR, Skoulidis F, Herter-Sprie GS, Buczkowski KA, Liu Y, Awad MM, Denning WL, et al. STK11/LKB1 deficiency promotes neutrophil recruitment and proinflammatory cytokine production to suppress T-cell activity in the lung tumor microenvironment. Cancer Res. 2016;76(5):999-1008.

73. Rojo de la Vega M, Chapman E, Zhang DD. NRF2 and the hallmarks of cancer. Cancer Cell. 2018:34(1):21-43.

74. Binkley MS, Jeon YJ, Nesselbush M, Moding EJ, Nabet BY, Almanza D, Kunder C, Stehr H, Yoo CH, Rhee S, et al. KEAP1/NFE2L2 mutations predict lung cancer radiation resistance that can be targeted by glutaminase inhibition. Cancer Discov. 2020;10(12):1826-41.

75. Jeong Y, Hellyer JA, Stehr H, Hoang NT, Niu X, Das M, Padda SK, Ramchandran K, Neal JW, Wakelee H, et al. Role of KEAP1/NFE2L2 mutations in the chemotherapeutic response of patients with non-small cell lung cancer. Clin Cancer Res. 2020;26(1):274-81.

76. Anagnostou V, Niknafs N, Marrone K, Bruhm DC, White JR, Naidoo J, Hummelink K, Monkhorst K, Lalezari F, Lanis M, et al. Multimodal genomic features predict outcome of immune checkpoint blockade in non-small-cell lung cancer. Nat Cancer. 2020;1 (1):99-111.

77. Passarelli A, Aieta M, Sgambato A, Gridelli C. Targeting immunometabolism mediated by CD73 pathway in EGFR-mutated non-small cell lung cancer: a new hope for overcoming immune resistance. Front Immunol. 2020;11:1479.

78. Concha-Benavente F, Ferris RL. Reversing EGFR mediated immunoescape by targeted monoclonal antibody therapy. Front Pharmacol. 2017;8:332.

79. Tanaka I, Morise M, Miyazawa A, Kodama Y, Tamiya Y, Gen S, Matsui A, Hase T, Hashimoto N, Sato M, et al. Potential benefits of bevacizumab combined with platinum-based chemotherapy in advanced nonsmall-cell lung cancer patients with EGFR mutation. Clin Lung Cancer. 2020;21(3):273-80.

80. Fukurnura D, Kloepper J, Amoozgar Z, Duda DG, Jain RK. Enhancing cancer immunotherapy using antiangiogenics: opportunities and challenges. Nat Rev Clin Oncol. 2018;15(5):325-40.

81. Shen J, Ju Z, Zhao W, Wang L, Peng Y, Ge Z, Nagel ZD, Zou J, Wang C, Kapoor P, et al. ARID1A deficiency promotes mutability and potentiates therapeutic antitumor immunity unleashed by immune checkpoint blockade. Nat Med. 2018;24(5):556-62.

82. Shin DS, Zaretsky JM, Escuin-Ordinas H, Garcia-Diaz A, Hu-Lieskovan S, Kalbasi A, Grasso CS, Hugo W, Sandoval S, Torrejon DY, et al. Primary resistance to PD-1 blockade mediated by JAK1/2 mutations. Cancer Discov. 2017:7(2):188-201.

83. Miao D, Margolis CA, Vokes NI, Liu D, Taylor-Weiner A, Wankowicz SM, Adeegbe D, Keliher D, Schilling B, Tracy A, et al. Genomic correlates of response to immune checkpoint blockade in microsatellite-stable solid tumors. Nat Genet. 2018;50(9):1271-81.

84. Schumacher TN, Schreiber RD. Neoantigens in cancer immunotherapy. Science. 2015;348(6230):69-74.

85. Cohen CJ, Gartner JJ, Horovitz-Fried M, Shamalov K, Trebska-McGowan K, Bliskovsky W, Parkhurst MR, Ankri C, Prickett TD, Crystal JS, et al. Isolation of neoantigen-specific T cells from tumor and peripheral lymphocytes. J Clin Invest. 2015;125(10):3981-91.

86. Chowell D, Morris LGT, Grigg CM, Weber JK, Samstein RM, Makarov V, Kuo F, Kendall SM, Requena D, Riaz N, et al. Patient HLA class I genotype influences cancer response to checkpoint blockade immunotherapy. Science. 2018;359(6375):582-7.

87. McGranahan N, Rosenthal R, Hiley CT, Rowan AJ, Watkins TBK, Wilson GA, Birkbak NJ, Veeriah S, Van Loo P, Herrero J, et al. Allelespecific HLA loss and immune escape in lung cancer evolution. Cell. 2017;171(6):1259-71. 
88. AbdulJabbar K, Raza SEA, Rosenthal R, Jamal-Hanjani M, Veeriah S, Akarca A, Lund T, Moore DA, Salgado R, Al Bakir M, et al. Geospatial immune variability illuminates differential evolution of lung adenocarcinoma. Nat Med. 2020;26(7):1054-62.

89. Morris SW, Kirstein MN, Valentine MB, Dittmer KG, Shapiro DN, Saltman DL, Look AT. Fusion of a kinase gene, ALK, to a nucleolar protein gene, NPM, in non-Hodgkin's lymphoma. Science. 1994;263(5151):1281-4.

90. Soda M, Choi YL, Enomoto M, Takada S, Yamashita Y, Ishikawa S, Fujiwara $\mathrm{S}$, Watanabe $\mathrm{H}$, Kurashina $\mathrm{K}$, Hatanaka $\mathrm{H}$, et al. Identification of the transforming EML4-ALK fusion gene in non-small-cell lung cancer. Nature. 2007:448(7153):561-6.

91. Shaw AT, Kim DW, Nakagawa K, Seto T, Crino L, Ahn MJ, De Pas T, Besse B, Solomon BJ, Blackhall F, et al. Crizotinib versus chemotherapy in advanced ALK-positive lung cancer. N Engl J Med. 2013;368(25):2385-94.

92. Solomon BJ, MokT, Kim DW, Wu YL, Nakagawa K, Mekhail T, Felip E, Cappuzzo F, Paolini J, Usari T, et al. First-line crizotinib versus chemotherapy in ALK-positive lung cancer. N Engl J Med. 2014;371(23):2167-77.

93. Ou SH. Crizotinib: a novel and first-in-class multitargeted tyrosine kinase inhibitor for the treatment of anaplastic lymphoma kinase rearranged non-small cell lung cancer and beyond. Drug Des Devel Ther. 2011;5:471-85.

94. Sakamoto H, Tsukaguchi T, Hiroshima S, Kodama T, Kobayashi T, Fukami TA, Oikawa N, Tsukuda T, Ishii N, Aoki Y. CH5424802, a selective ALK inhibitor capable of blocking the resistant gatekeeper mutant. Cancer Cell. 2011;19(5):679-90.

95. Hida T, Nokihara H, Kondo M, Kim YH, Azuma K, Seto T, Takiguchi Y, Nishio M, Yoshioka H, Imamura F, et al. Alectinib versus crizotinib in patients with ALK-positive non-small-cell lung cancer (J-ALEX): an open-label, randomised phase 3 trial. Lancet. 2017;390(10089):29-39.

96. Peters S, Camidge DR, Shaw AT, Gadgeel S, Ahn JS, Kim DW, Ou SI, Perol M, Dziadziuszko R, Rosell R, et al. Alectinib versus crizotinib in untreated ALK-positive non-small-cell lung cancer. N Engl J Med. 2017;377(9):829-38.

97. Zhou C, Kim SW, Reungwetwattana T, Zhou J, Zhang Y, He J, Yang JJ Cheng $\mathrm{Y}$, Lee $\mathrm{SH}$, Bu L, et al. Alectinib versus crizotinib in untreated Asian patients with anaplastic lymphoma kinase-positive non-small-cell lung cancer (ALESIA): a randomised phase 3 study. Lancet Respir Med. 2019;7(5):437-46.

98. Harrer S, Shah P, Antony B, Hu J. Artificial intelligence for clinical trial design. Trends Pharmacol Sci. 2019;40(8):577-91.

99. Leonetti A, Sharma S, Minari R, Perego P, Giovannetti E, Tiseo M. Resistance mechanisms to osimertinib in EGFR-mutated non-small cell lung cancer. Br J Cancer. 2019;121(9):725-37.

100. Petak I, Kamal M, Dirner A, Bieche I, Doczi R, Mariani O, Filotas P, Salomon A, Vodicska B, Servois V, et al. A computational method for prioritizing targeted therapies in precision oncology: performance analysis in the SHIVA01 trial. NPJ Precis Oncol. 2021;5(1):59.

101. Abbasi M, Amanlou M, Aghaei M, Hassanzadeh F, Sadeghi-Aliabadi H. Identification of new Hsp90 inhibitors: structure based virtual screening, molecular dynamic simulation, synthesis and biological evaluation. Anticancer Agents Med Chem. 2021. https://doi.org/10.2174/18715 20621666210201101818.

102. Kilchmann F, Marcaida MJ, Kotak S, Schick T, Boss SD, Awale M, Gonczy $P$, Reymond JL. Discovery of a selective aurora A kinase inhibitor by virtual screening. J Med Chem. 2016;59(15):7188-211.

\section{Publisher's Note}

Springer Nature remains neutral with regard to jurisdictional claims in published maps and institutional affiliations.
Ready to submit your research? Choose BMC and benefit from:

- fast, convenient online submission

- thorough peer review by experienced researchers in your field

- rapid publication on acceptance

- support for research data, including large and complex data types

- gold Open Access which fosters wider collaboration and increased citations

- maximum visibility for your research: over 100M website views per year

At BMC, research is always in progress.

Learn more biomedcentral.com/submissions 